\title{
La inseguridad subjetiva como mediadora del bienestar social y clima emocional
}

Marcela Muratori ${ }^{1}$ y Elena Zubieta ${ }^{2}$

\section{Artículo}

Material original autorizado para la publicación en la revista Psicodebate. Facultad de Ciencias Sociales. Universidad de Palermo.

Recibido 27-07-2016 | Aceptado 28-08-2016

\section{Resumen}

Múltiples estudios revelan que no sólo el impacto de hechos negativos tales como haber sido testigo o víctima de algún delito sino incluso una elevada percepción de inseguridad, entendida esta como riesgo percibido y miedo al delito, tienen importantes consecuencias sociales y condicionan, de alguna manera, la calidad de vida e integración social de las personas. En el contexto de un alto sentimiento de desconfianza y aumento de la inseguridad, esta investigación plantea analizar la relación entre los factores asociados a la inseguridad ciudadana y la percepción del contexto social. Para ello, se llevó a cabo un estudio de naturaleza correlacional, de diseño no experimental. La muestra, no probabilística intencional, estuvo compuesta por 516 estudiantes universitarios (44\% mujeres; 56\% hombres; $M_{E d a d}=23.04 ; D E=4.08$ ), residentes en la Ciudad de Buenos Aires y alrededores. Los datos revelan que, a pesar de los bajos niveles de miedo al delito, los participantes perciben una alta probabilidad de volverse víctimas de delitos en el futuro cercano, razón por la cual adoptarían conductas de auto-protección. Asimismo, la mayoría de los participantes admite haber sido víctima directa de algún delito, porcentaje aún mayor con respecto a la victimización indirecta. Si bien los resultados muestran niveles relativamente altos de bienestar social, prevalece un clima emocional negativo. Se observan relaciones entre el bienestar social, la percepción del clima emocional, la victimización, el miedo al delito y el riesgo percibido. Más aún, estos factores junto a las conductas de auto-protección median la relación entre la victimización y algunas de las dimensiones de la percepción del contexto social. Se concluye que, más allá de la inseguridad objetiva, que debe ser controlada

1 UBA, CONICET, Instituto de Investigaciones, Facultad de Psicología - Argentina

2 UBA, CONICET, Instituto de Investigaciones, Facultad de Psicología - Argentina 
e idealmente reducida, es necesario profundizar en el estudio de la inseguridad subjetiva, dado sus efectos en la calidad de vida de las personas.

Palabras Clave: inseguridad ciudadana, riesgo percibido, miedo al delito, contexto social, bienestar social, clima emocional.

\section{Subjective insecurity as a mediator of social well-being and emotional climate}

\section{Abstract}

Multiple studies reveal that not only the impact of negative factors such as being a witness or victim of a crime but a high perception of insecurity, understood as perceived risk and fear of crime, have important social consequences and condition, somehow, people's quality of life and social integration. In the context of a high sense of mistrust and the increasing insecurity, this research analyzes the relationship between the factors associated with insecurity and the perception of social context. To this end, a correlational, non-experimental design study was conducted. The sample, not probabilistic intentional, consisted of 516 college students ( $44 \%$ women; $56 \%$ men; $M_{A g e}=23.04 ; S D=4.08$ ), residents of the City of Buenos Aires and surroundings. The data show that, despite low levels of fear of crime, participants perceive a high probability of becoming crime victims in the near future, reason why they would adopt self-protective behaviors. In addition, most of the participants admitted having been a direct victim of a crime, even higher percentage with respect to indirect victimization. While the results show relatively high levels of social well-being, a negative emotional climate prevails. Relations between social well-being, the perception of emotional climate, victimization, fear of crime and perceived risk are observed. Moreover, these factors along with self-protective behaviors mediate the relationship between victimization and some of the dimensions of perceived social context. It is concluded that, beyond the objective insecurity, which should be controlled and ideally reduced, it is necessary to deepen the study of subjective insecurity, given its effects on the quality of life of people.

Keywords: subjective insecurity, perceived risk, fear of crime, social context, social well-being, emotional climate. 
En Argentina, como en gran parte de Latinoamérica, la inseguridad es un problema de relevancia social, configurándose como el centro de las preocupaciones públicas en el que compite sólo con la problemática socioeconómica (Föhrig, 2006; Kessler, 2012).

La seguridad, en tanto condición humana, es considerada una de las necesidades básicas por excelencia, siendo esencial para el bienestar y desarrollo de la persona (Maslow, 1954/1987; Schwartz, 2001). Se ubica así dentro de la categoría de necesidades psicológicas, considerándose como un impulso del organismo que activa y orienta la conducta hacia metas que, al ser satisfechas, contribuyen no sólo a la supervivencia y bienestar, sino también a la salud (Páez, Morales, \& Fernández, 2007). Es por esta razón que el percibir seguridad en la vivienda, el barrio, la ciudad y la sociedad en su conjunto es un requisito fundamental para el bienestar de las personas (Franc, Prizmic-Larsen, \& Kaliterna Lipovcan, 2012).

En este marco, hechos negativos como ser testigos de sucesos violentos o el haber sufrido experiencias traumáticas y de violencia tienen efectos devastadores tanto sobre las personas afectadas como sobre sus familiares (Chía Chávez, Bilbao, Páez, Iraurgi, \& Beristain, 2011). Abundante literatura da cuenta de los efectos del crimen y la victimización sobre distintos aspectos de la vida de las personas, que van desde daños físicos y económicos (por la sustracción objetiva de los bienes, pérdida de tiempo laboral y gastos médicos por heridas ocasionadas) hasta morales y psicológicos (Freeman, 1999). Además, muchos estudios se han centrado en las consecuencias de la victimización sobre el bienestar de las personas (Cohen, 2008; Hanslmaier, 2013; Hanson, Sawyer, Begle, \& Hubel, 2010; Kuroki, 2013; Staubli, Killias, \& Frey, 2014), evidenciando que tanto el haber sido víctima directa como indirecta tiene importantes implicancias en la salud de las personas, dado que en ambos casos disminuye la percepción de calidad de vida, la satisfacción y la felicidad. Por lo tanto, la victimización criminal ha demostrado ser un predictor significativo en el bienestar. Junto a la victimización, muchas investigaciones han mostrado interés por el efecto del miedo al delito sobre la salud física y mental de las personas (Davies \& Hinks, 2010; Franc et al., 2012; Jacskon \& Stafford, 2009; Morrall, Marshall, Pattison, \& Macdonald, 2010; Sulemana, 2015), revelando que este afecta el bienestar psicológico de víctimas y no víctimas de delitos. De hecho, en algunos casos el miedo al delito y la preocupación por la seguridad personal pueden tener un mayor impacto en el bienestar y la satisfacción con la vida que la victimización misma. Para Pegoraro (2002), es indiscutible el hecho de que estas percepciones están incorporadas a la cotidianeidad de la vida moderna y conllevan consecuencias para la vida democrática, más allá de la realidad objetiva del fenómeno.

Hay consenso entre quienes estudian la temática de que, para reducir el miedo al delito, el desafío consiste en reducir el crimen (Ferraro, 1995). Por ello, gran 
parte del interés académico, social y político sobre el miedo al crimen se ha centrado en la relación entre el crimen y el miedo, y en el impacto negativo que genera en individuos y en comunidades (Jackson, 2009; Vozmediano, Vergara, \& San Juan, 2010). Sin embargo, distintos estudios han demostrado que no existe una correspondencia entre las tasas de delito y la percepción subjetiva de los ciudadanos respecto a la inseguridad (Kessler, 2009; Smulovitz, 2006; Vozmediano, 2010). Además, como plantean Míguez e Isla (2010), no es sólo el incremento de la frecuencia de delitos lo que hace que se propague socialmente la percepción de inseguridad, sino que hay otros factores asociados a ella, como por ejemplo los medios de comunicación o los niveles de confianza que generan las agencias del Estado encargadas de controlar la actividad delictiva y de proponer políticas que la prevengan, por nombrar sólo algunos. Moreno (2014) considera imprescindible evaluar el problema de la inseguridad desde su doble naturaleza: el haber sido víctima de un hecho delictivo y la percepción de inseguridad, ya que ambas facetas tienen importantes consecuencias sociales y condicionan la calidad de vida e integración social de las personas.

El análisis de la percepción de inseguridad no permite la adopción de una mirada estática, ya que se inscribe en un contexto dinámico socio-político, psicológico-social y geo-espacial determinado (Farral \& Lee, 2009). Por lo tanto, este estudio se enmarca en una perspectiva psicosocial que hace foco en el vínculo que los individuos establecen con el entorno, en su confianza en el futuro de la sociedad y en la percepción sobre la capacidad de este para proveerles bienestar y poder realizarse como personas. Hoy en día, y con especial énfasis en el contexto argentino, numerosos estudios revelan cierto malestar en los individuos respecto de la capacidad de la sociedad para darles seguridad, sentido de confianza, de pertenencia y de un propósito común (Delfino, 2009; Kessler, 2009; Míguez \& Isla, 2010; Moreno, 2014; Muratori, Fernández, Bombelli, \& Zubieta, 2014; Zubieta, Muratori, \& Mele, 2012). Esto, acompañado de las cifras objetivas suministradas por organismos gubernamentales y no gubernamentales acerca de los hechos delictivos y la desconfianza en las instituciones, los discursos políticos, las encuestas de victimización, la transmisión de información de los medios de comunicación, las manifestaciones y demandas populares, ha llevado a que temas como la percepción de riesgo y el miedo al delito se vuelvan objetos de estudio de las ciencias sociales por su actualidad y urgencia. Así, a la investigación que aquí se presenta subyace la necesidad de reflexionar sobre la incidencia que la inseguridad subjetiva, en términos de miedo al delito y percepción de riesgo, más allá de la victimización real y objetiva, tiene en la percepción del contexto social y del clima social emocional, y en su impacto en los niveles de bienestar social y de calidad de vida de los individuos. El trabajo propone contribuir a la comprensión 
del fenómeno cubriendo un área de vacancia a nivel local: el del análisis de los aspectos psicosociales de la inseguridad y la percepción del contexto social.

\section{Método}

\section{Tipo de estudio}

Se desarrolló un estudio de naturaleza descriptivo-correlacional, de diseño no experimental transversal, con estudiantes universitarios de ambos sexos como unidad de análisis.

\section{Participantes}

La muestra, no probabilística intencional, estuvo compuesta por 516 estudiantes universitarios, los cuales representaban dos contextos diferenciados en lo que hace al grado de implicación con la seguridad: civil $(n=267)$ y militar $(n=249)$. El rango de edad fue de entre 18 y 40 años, con una media de 23.04 años $(D E=4.08)$. El 56\% eran hombres y el 44\% mujeres. El 30.5\% habitaba en la Ciudad Autónoma de Buenos Aires, el 14.4\% en la zona norte del conurbano bonaerense, el $33.5 \%$ en la zona oeste y el $21.6 \%$ en la zona sur.

\section{Materiales}

Para el presente estudio, se tomaron en consideración las siguientes escalas:

1- Percepción de riesgo. Consta de dos preguntas acerca de la percepción de riesgo en niveles de referencia personal y general. Los participantes debían responder cuán probable cree que en el próximo año pueda convertirse en víctima de un delito versus cuán probable cree que en el próximo año un habitante de la ciudad sea víctima de algún delito $(1=$ nada probable a $10=$ muy probable $)$.

2- Escala de Miedo al Delito (Vozmediano, 2010). Sobre una lista de 12 delitos que atentan contra la seguridad, se les pidió a los participantes que respondieran a la pregunta "¿Con qué frecuencia ha sentido temor o inquietud por la posibilidad de ser víctima de estos delitos?" $(1=$ Nunca a $5=$ Siempre $)$. Los índices de fiabilidad fueron altos $(\alpha=0.92)$.

3 - Conductas de auto-protección frente a la percepción de peligro. Se construyó una escala ad hoc a partir de la revisión de la Escala de Afrontamiento frente a la percepción de peligro (Espinosa, 2006, como se citó en Rottenbacher de Rojas, Amaya López, Genna Miyahira, \& Pulache Páez, 2009). En 19 ítems, se evalúa la intención de las personas de evitar una posible exposición al peligro. Los participantes debían responder con qué frecuencia realizan una serie de conductas $(1=$ Nunca hasta $5=$ Siempre $)$. La confiabilidad general fue alta $(\alpha=.85)$.

4- Escala de victimización. Para medir la frecuencia de victimización directa 
se construyó una escala sobre la base de la escala de victimización empleada por Vozmediano (2010). En ella se presentaron los mismos delitos presentados en la Escala de Miedo al Delito, a excepción del ítem Homicidio. Los participantes debían responder a la pregunta: Durante el último año, ¿ha sido víctima en alguna ocasión de estos delitos? $(0=$ Nunca, $1=1$ o 2 veces y $2=3$ o más $)$.

Para evaluar la victimización indirecta se incluyó un ítem donde se preguntaba al participante si fue testigo de algún delito en el último año $(0=$ Nunca, $1=1$ o 2 veces y $2=3$ o más). Además, se preguntó si amigos, familiares o allegados fueron víctimas de algún delito $(1=n o, 2=s i)$. Finalmente, se construyó una variable que combinara las tres categorías: víctima directa (si/no), víctima indirecta como testigo (si/no), víctima indirecta por conocimiento de familiar y/o amigo (si/no), dando origen a una variable de exposición a la violencia con cuatros valores: $1=$ no afectado; 2 = víctima indirecta, 3 = víctima directa, 4 = víctima directa e indirecta.

5- Escala de Bienestar Social (Keyes, 1998; Muratori \& Zubieta, 2016). En 17 ítems, con puntuación de tipo Likert de 1 (totalmente en desacuerdo) a 5 (totalmente de acuerdo), evalúa la percepción que las personas tienen sobre cinco aspectos del entorno social que facilitan su bienestar. Las dimensiones son: a) Integración Social o sentirse parte de la comunidad, sentirse que se pertenece, se tiene apoyo y se comparten cosas con el colectivo ("Me siento cercano a otra gente"), facilitando la satisfacción de las necesidades de apego, afiliación y pertenencia (3 ítems: $\alpha=.633$ ); b) Aceptación Social o que la persona tiene una actitud positiva hacia los otros en general ("Creo que las personas sólo piensan en sí mismas"-ítem inverso). Implica percibir que las relaciones con otros permiten auto-aceptarse y obtener autoestima (4 ítems: $\alpha=.800$ ); c) Contribución Social o sentimiento de tener algo positivo que dar a la sociedad y que las actividades que uno hace son valoradas ("Pienso que lo que hago es importante para la sociedad") (3 ítems: $\alpha=.767)$; d) Actualización Social o creer que el mundo social se desarrolla o puede desarrollarse para mejor ("Para mí el progreso social es algo que no existe"- ítem inverso). Se asocia con percibir que el entorno permite el crecimiento personal (3 ítems: $\alpha=.618$ ); y e) Coherencia Social o creencia de que el mundo es predecible, inteligible y lógico ("No entiendo lo que está pasando en el mundo"-ítem inverso) (4 ítems: $\alpha=.603$ ). El coeficiente de fiabilidad de esta escala fue muy satisfactorio $(\alpha=.791)$.

6- Escala de clima emocional (Páez et al., 1997). Esta escala evalúa la percepción de emociones positivas (alegría, esperanza, solidaridad) y los procesos sociales que las refuerzan (confianza en las instituciones, tranquilidad para hablar), así como las emociones negativas dominantes en el clima social o en la interacción cotidiana (tristeza, miedo y enojo). Está compuesta por nueve ítems con un continuo de respuesta de 1 (nada) a 5 (mucho). Para analizar su estructura se recurrió a un análisis factorial de componentes principales con rotación 
Varimax. Con una medida de adecuación muestral KMO de .814, se corroboran las dos dimensiones subyacentes planteadas por Techio et al. (2011), las cuales explican el $54.87 \%$ de la varianza. Estas son: a) Clima emocional positivo (6 ítems: $\alpha=.77)$ : explica el $38.45 \%$ de la varianza; b) Clima emocional negativo (3 ítems: $\alpha=.75$ ): explica el $16.42 \%$ de la varianza.

\section{Procedimiento y análisis de datos}

La administración del cuestionario se realizó de forma colectiva entre finales del año 2012 y principios del año 2014. Se contactó a profesores universitarios que cedieron una hora de sus clases y los estudiantes fueron invitados a participar voluntariamente y de forma anónima, garantizándole la confidencialidad de sus respuestas, solicitándoles su consentimiento informado.

Con respecto a los análisis de datos, para los objetivos descriptivos se efectuaron análisis de frecuencias, reportándose los porcentajes, la media y el desvío estándar, según el caso. A fin de analizar la relación entre las variables se realizaron correlaciones y análisis mediacionales. Con el objetivo de contrastar la hipótesis de mediación, se utilizó el macro de SPSS con el método bootstrap para efectos indirectos, que permite estimar el efecto indirecto, los errores estándar y los intervalos de confianza derivados de la distribución bootstrap. E1 efecto indirecto resulta significativo si el intervalo de confianza no pasa por el cero. Asimismo, se propone que el efecto total no necesariamente tiene que ser significativo para que haya mediación, puesto que lo importante es la diferencia absoluta entre el efecto total y el indirecto (Preacher \& Hayes, 2008). Al realizar este análisis se estableció el grado de victimización como variable predictora, los dos tipos de percepción de inseguridad (miedo al delito y riesgo percibido) y las conductas de auto-protección fueron analizadas como mediadoras y los diferentes indicadores de percepción del contexto social como variables dependientes del modelo. Los análisis fueron realizados controlando el género y tipo de muestra.

\section{Resultados}

\section{Resultados descriptivos}

\section{Percepción de riesgo}

Los participantes consideran que es altamente probable que ellos mismos se conviertan en víctimas de algún delito en el próximo año $(\mathrm{M}=6.01 ; \mathrm{DE}=$ 2.51), aunque estos valores son significativamente superiores en lo referido a un habitante de la ciudad $(M=8.55 ; \mathrm{DE}=2.07)$ y no a sí mismos, $\mathrm{t}(507)=-20.51$; $p<.001$. Este dato corrobora la ilusión de invulnerabilidad a la victimización, 
producto de la comparación social en las que las personas estiman su propio riesgo en niveles menores al de los otros.

\section{Miedo al delito}

Los participantes presentan puntuaciones relativamente bajas en miedo al delito, siendo el robo en la calle el único delito que supera la media teórica $\left(M_{\text {teórica }}=3\right)$ (ver tabla 1).

Tabla 1.

Puntuaciones y desviaciones típicas de miedo al delito.

\begin{tabular}{lcc}
\hline & M & DE \\
\hline Miedo al delito & 2.48 & 0.94 \\
Robo en la calle & 3.40 & 1.29 \\
Robo en su casa cuando no hay nadie & 2.82 & 1.36 \\
Robo o daños de/en vehículo & 2.73 & 1.45 \\
Robo en su casa, cuando hay alguien dentro & 2.64 & 1.29 \\
Robo/fraude con tarjetas de crédito & 2.47 & 1.30 \\
Secuestro/ser retenido contra su voluntad & 2.41 & 1.33 \\
Agresión sexual & 2.25 & 1.36 \\
Homicidio & 2.10 & 1.31 \\
Víctima de exceso de violencia de fuerzas públicas & 2.00 & 1.92 \\
Atentado terrorista & 1.58 & 1.05 \\
Agresión física & 2.80 & 1.29 \\
Acoso/amenazas/hostigamiento verbal & 2.56 & 1.34 \\
\hline
\end{tabular}

\section{Conductas de auto-protección frente a la percepción de peligro}

Los participantes presentan conductas de protección personal prudentes $(M=$ 3.18; $D E=0.77)$, dejando de lado las medidas extremas $(M=1.66 ; D E=0.74)$. Las conductas más frecuentes son: cerrar bien las ventanas y puertas cuando salen de la casa $(M=4.02 ; D E=1.22)$, trasladarse por zonas y rutas transitadas por mucha gente $(M=3.67 ; D E=1.09)$, evitar sacar plata de los cajeros automáticos por la noche $(M=3.41 ; D E=1.39)$ y estar atentos a si alguien los sigue $(M=3.40$; $D E=1.26)$. Por el contrario, las conductas más infrecuentes son las de evitar salir a la calle de día $(M=1.54 ; D E=.96)$, evitar salir a caminar por la calle de día $(M=$ $1.56 ; D E=.93)$, evitar salir a restaurantes $(M=1.64 ; D E=1.01)$ y llevar un arma u objeto defensivo cuando sale de su casa $(M=1.64 ; D E=1.12)$. 


\section{Victimización}

Respecto a la victimización, el $56.7 \%$ de los participantes afirma haber sido víctima directa de al menos un delito. Cabe destacar que el $79.2 \%$ de los participantes tiene un amigo, familiar o allegado que ha sido víctima de algún delito y que el 35.4\% ha sido testigo de algún delito, por lo que el porcentaje de victimización indirecta es también muy alto. Asimismo, es bajo el porcentaje de participantes (12.3\%) que no ha sido víctima en ninguna de sus formas, de manera directa o indirecta.

\section{Percepción del contexto social}

\section{Bienestar social}

Como puede observarse en la tabla 2, los participantes presentan niveles relativamente altos en bienestar social, siendo que la puntuación más alta se encuentra en la dimensión contribución y la más baja en aceptación. Esto indica que el aspecto más óptimo respecto a la valoración que se hace de la relación con el entorno se vincula con el sentimiento de utilidad por parte de los participantes, que sienten que son miembros vitales de la sociedad, se sienten eficaces y aportan al bien común. Asimismo, el aspecto levemente deficitario es la baja confianza que se vivencia respecto de los otros, de las actitudes positivas entre las personas y de la atribución de honestidad, bondad, amabilidad y capacidad.

Tabla 2.

Puntuaciones medias y desviaciones típicas en bienestar social

\begin{tabular}{lcc}
\hline Dimensiones & $M$ & $D E$ \\
\hline Integración social & 4.03 & 0.69 \\
Aceptación social & 3.05 & 0.86 \\
Contribución social & 4.22 & 0.83 \\
Actualización social & 3.72 & 0.88 \\
Coherencia social & 3.82 & 0.77 \\
\hline
\end{tabular}

Nota. Continuo de respuesta de $1=$ totalmente en desacuerdo a $5=$ totalmente de acuerdo.

\section{Clima Emocional}

En lo que hace a la percepción del clima emocional, es decir, a la emoción que se percibe como predominante en el ambiente (ver tabla 3 ), los participantes experimentan un alto clima emocional negativo, por sobre el clima emocional positivo. De manera coherente, al nivel de las emociones sentidas, predominan el enojo, hostilidad y agresión entre la gente, miedo y ansiedad, tristeza y pasividad. En las emociones y vivencias positivas, las puntuaciones están todas por debajo de 3 (media teórica), lo que indica que 
son las que los sujetos perciben como menos frecuentes en el entorno. Según reportan Páez y Asún (1994), el perfil emocional colectivo negativo se asocia, en términos de conductas sociales, a un menor altruismo, menor cooperación y mayor conflicto grupal.

Tabla 3.

Puntuaciones medias y desviaciones típicas de los ítems de la escala de clima emocional

\begin{tabular}{lcc}
\hline Ítems & $M$ & $D E$ \\
\hline El clima o ambiente general afectivo de su país es muy bueno & 2.56 & 1.03 \\
El ambiente/clima es de: & & \\
Esperanza & 2.36 & 0.92 \\
Solidario/de ayuda mutua & 2.88 & 0.98 \\
Confianza en las instituciones & 2.19 & 0.92 \\
Miedo/ansiedad & 3.66 & 1.07 \\
Enojo/hostilidad/agresividad entre las personas & 3.70 & 1.06 \\
Tristeza/pasividad/ bajo estado anímico & 3.21 & 1.08 \\
Alegría/confianza/contento & 2.63 & 0.95 \\
Tranquilidad para hablar & 2.68 & 1.10 \\
\hline Clima negativo & 3.53 & 0.87 \\
Clima positivo & 2.55 & 0.66 \\
\hline
\end{tabular}

Nota. Continuo de respuesta de $1=$ nada a $5=$ mucho.

Relación entre la percepción del contexto social y las variables asociadas a la inseguridad: análisis mediacionales

Como puede observarse en la tabla 4, la percepción del contexto social varía en función de los indicadores de inseguridad, en especial en lo que hace a los aspectos más sociales y macro del entorno; esto es, del clima emocional. Cabe resaltar que son los aspectos conductuales de la inseguridad ciudadana los que más se relacionan con la percepción del contexto social.

En lo que hace al bienestar social, una mayor percepción de riesgo se asocia de manera negativa a la actualización social, mientras el miedo al delito se asocia de manera negativa a la aceptación. Es decir, una alta percepción de inseguridad reduce el optimismo respecto al futuro de la sociedad y las actitudes positivas hacia los otros. Por su parte, las conductas de auto-protección se asocian de manera negativa a todas las dimensiones del bienestar social, a excepción de la contribución.

Respecto al clima emocional, el clima positivo se relaciona negativa y significativamente a los distintos indicadores de la inseguridad, mientras el clima negativo lo hace en sentido inverso. 
Tabla 4.

Correlaciones entre percepción de contexto social e inseguridad ciudadana.

\begin{tabular}{lcccc}
\hline & $\begin{array}{c}\text { Riesgo } \\
\text { percibido }\end{array}$ & $\begin{array}{c}\text { Miedo al } \\
\text { delito }\end{array}$ & Auto-protección & Victimización \\
\hline Integración social & -.06 & .02 & $.09^{\star}$ & -.03 \\
Aceptación social & -.08 & $-.10^{\star}$ & $-.21^{\star \star}$ & .00 \\
Contribución social & .02 & .04 & -.02 & -.07 \\
Actualización social & $-.16^{\star \star}$ & $-.09^{\star}$ & $-.13^{\star \star}$ & -.00 \\
Coherencia social & .03 & -.04 & $-.15^{\star \star}$ & .02 \\
\hline Clima positivo & $-.24^{\star \star}$ & $-.23^{\star \star}$ & $-.22^{\star \star}$ & $-.16^{\star \star}$ \\
Clima negativo & $.24^{\star \star}$ & $.27^{\star \star}$ & $.24^{\star \star}$ & $.21^{\star \star}$ \\
\hline${ }^{*} p<0.05,{ }^{* \star} p<0.01$ & & & &
\end{tabular}

Finalmente, con el objetivo de indagar en el sentido de las relaciones expuestas, se hipotetizó que tanto las percepciones de inseguridad como las conductas de auto-protección median en el efecto de los niveles de victimización sobre el bienestar social y clima emocional percibido.

En primer lugar, se analizan los efectos mediadores del miedo al delito y el riesgo percibido sobre la asociación entre la victimización y los distintos indicadores de la percepción del contexto social. A continuación se presentan los modelos de mediación en los cuales los efectos indirectos son estadísticamente significativos.

Todos los análisis de regresión indican que la victimización es un predictor significativo de la percepción de inseguridad, por lo que la mayor exposición al delito se asocia a un mayor miedo y a una mayor percepción de riesgo. En lo que hace a la asociación entre la victimización y el miedo al delito, el coeficiente de regresión es de .11 para todos los casos. Respecto a la relación entre victimización y percepción de riesgo, el coeficiente varía entre .18 a .21, según las variables analizadas.

En cuanto al bienestar social, sólo se encuentran efectos de mediación significativos en relación con la actualización social (ver figura 1). En este caso, sólo el riesgo percibido se asocia significativamente a la actualización social. La victimización no es un predictor significativo de la actualización social, aun cuando se controla el miedo al delito y el riesgo percibido, siendo el efecto directo no significativo. Sin embargo, la prueba de efecto indirecto basada en el procedimiento bootstrap de Preacher y Hayes (2008) es significativa a través del riesgo percibido $\left(B_{\text {efecto indirecto }}=-0.01\right.$; Boot $D E=0.00$; IC95\% [-0.0217. -0.0037]). Por ende, una mayor victimización está asociada a una mayor percepción de riesgo en comparación con los menos victimizados, lo que lleva a una peor evaluación acerca del potencial de crecimiento y desarrollo de la sociedad. 


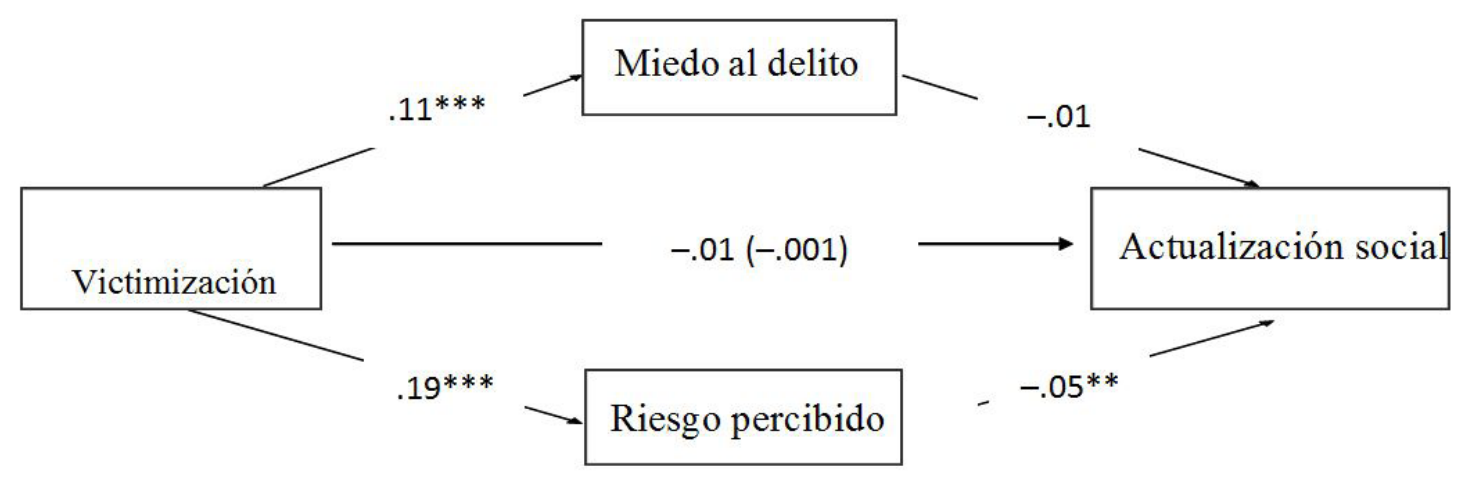

Figura 1. Efecto de victimización sobre la actualización social, mediado por el miedo al delito y el riesgo percibido. En la figura se muestran los coeficientes de regresión no estandarizados. Además, se muestra el coeficiente del efecto total y, entre paréntesis, el coeficiente del efecto directo de la victimización sobre la actualización social. ${ }^{\star} p<.05 .{ }^{\star \star} p<.01 .{ }^{\star \star \star} p<.001$.

Con respecto al clima emocional, se obtienen efectos de mediación significativos tanto para el clima emocional positivo como para el negativo. Como muestran las figuras 2 y 3 , el efecto total de la exposición al delito sobre el clima emocional es significativo y negativo respecto al clima positivo, mientras que significativo y positivo sobre el clima negativo. Esto es, una mayor victimización reduce la percepción del clima emocional positivo a la vez que aumenta la afectividad negativa. Estos efectos son menores al incluir en el modelo a las percepciones de inseguridad como mediadores, confirmando la hipótesis acerca de los efectos indirectos de la inseguridad objetiva mediada por la subjetiva (miedo al delito y riesgo percibido). Por un lado, los análisis de boostrap muestran un efecto indirecto de la victimización sobre el clima positivo mediado por el miedo al delito $\left(B_{\text {efecto indirecto }}=-0.01\right.$; Boot $D E=0.00$; IC95\% $[-0.0177$. -0.015$])$, y por la percepción de riesgo $\left(B_{\text {efecto indirecto }}=-0.01\right.$; Boot $D E=$ 0.00; IC95\% [-0.0187. -0.0043]). Así, los efectos negativos de la victimización sobre la percepción del clima positivo se explican, en parte, por un mayor miedo al delito y una mayor percepción de riesgo en quienes mayor exposición al delito exhiben, en comparación con los menos expuestos o no afectados. Se sostiene que esto sucede, en parte, debido a que el coeficiente de la victimización en el clima positivo disminuye cuando se controla el miedo y la percepción de riesgo pero, aunque menor, sigue siendo significativo. Por otro lado, los análisis de boostrap muestran un efecto indirecto de la victimización sobre el clima negativo mediado por el miedo al delito $\left(B_{\text {efecto indirecto }}=0.02\right.$; Boot $D E=0.01$; IC95\% $[0.0088$. $0.0338])$ y el riesgo percibido $\left(B_{\text {efecto indirecto }}=0.01\right.$; Boot $D E=0.00 ;$ IC95\% $[0.0025$. $0.0189])$. En otras palabras, el efecto de haber sido víctima directa e indirecta 
de algún delito sobre la percepción del clima emocional negativo se explica a través de la inseguridad subjetiva. La mediación es parcial, ya que el coeficiente de la victimización en el clima negativo disminuye cuando se controla el miedo y la percepción de riesgo pero, aunque menor, sigue siendo significativo - como ocurre en el caso del clima positivo.

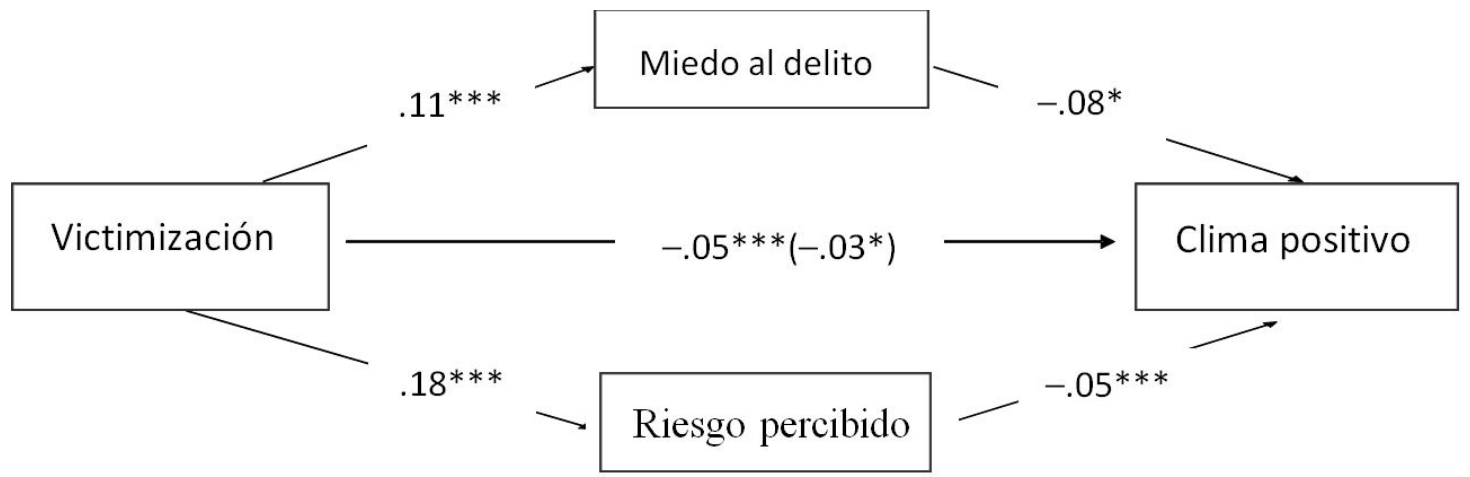

Figura 2. Efecto de la victimización sobre el clima emocional positivo, mediado por el miedo al delito y la percepción de riesgo. En la figura se muestran los coeficientes de regresión no estandarizados. Además, se muestra el coeficiente del efecto total y, entre paréntesis, el coeficiente del efecto directo de la victimización sobre la actualización social.

${ }^{\star} p<.05 .{ }^{* \star} p<.01 .{ }^{* \star *} p<.001$.

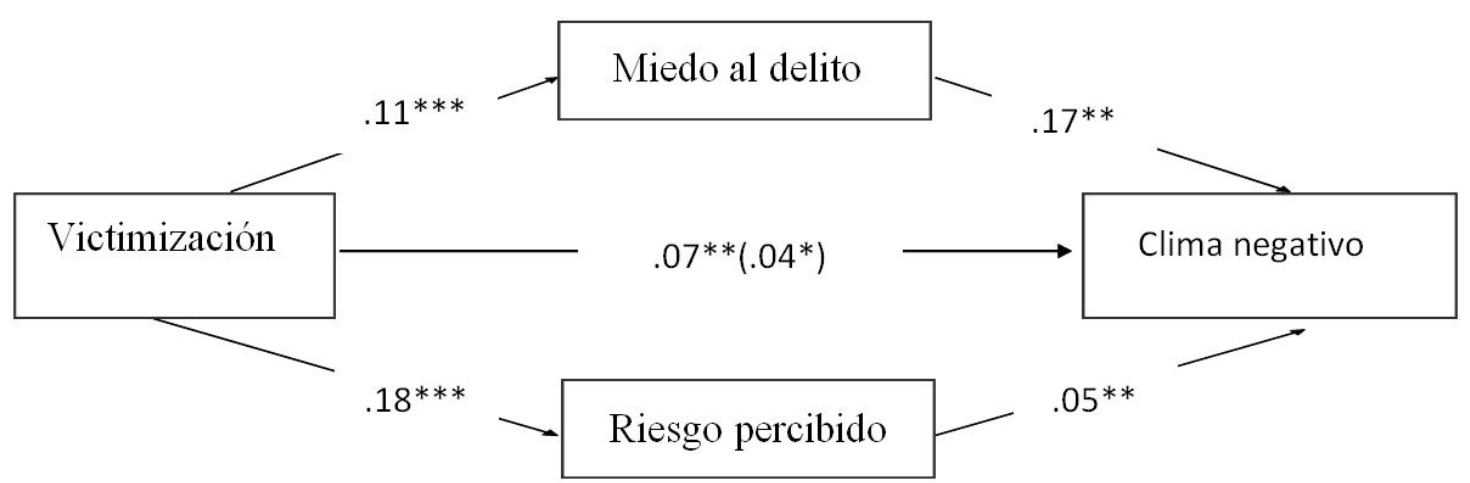

Figura 3. Efecto de la victimización sobre el clima emocional negativo, mediado por el miedo al delito y la percepción de riesgo. En la figura se muestran los coeficientes de regresión no estandarizados. Además, se muestra el coeficiente del efecto total y, entre paréntesis, el coeficiente del efecto directo de la victimización sobre la actualización social.

$$
{ }^{\star} p<.05 .{ }^{\star \star} p<.01 .{ }^{\star \star \star} p<.001 .
$$

Finalmente, se evalúan las conductas de auto-protección como mediadores entre la relación de la victimización y la percepción del contexto social, en tanto que estas no sólo son indicadores de la inseguridad ciudadana sino que también expresan de manera manifiesta las distintas percepciones de los participantes. 
Sólo se exhiben los modelos de mediación en los que los efectos indirectos resultaron estadísticamente significativos.

Primero, la victimización es un predictor significativo de las conductas de autoprotección donde, a mayor exposición al delito, mayor es la frecuencia de conductas de auto-protección. El coeficiente de regresión es de .05 para todos los casos.

Como se puede apreciar en la figura 4, las conductas de auto-protección se asocian significativamente a la integración social. Finalmente, la victimización no surge como un predictor significativo de la integración, aun cuando se controlan las conductas de auto-protección, siendo el efecto directo no significativo en estos modelos. No obstante, las pruebas de efecto indirecto basadas en el procedimiento bootstrap son significativas $\left(B_{\text {efecto indirecto }}=0.005\right.$; Boot $D E=0.00$; IC95\% [0.003. 0.0128]). Este efecto puede considerarse un efecto de supresión, ya que las conductas de auto-protección aumentan la validez predictiva de la variable victimización sobre la integración por su inclusión en la regresión, es decir, se cultiva el sentimiento de pertenencia y se construyen lazos sociales más fuertes. Esta validez predictiva se evalúa a través del coeficiente de regresión aumentado por la inclusión del supresor. Es decir, las diferencias en los niveles de victimización en la integración social en términos de coeficiente de regresión aumentan cuando se introduce la variable conductas de auto-protección (Conger, 1974).

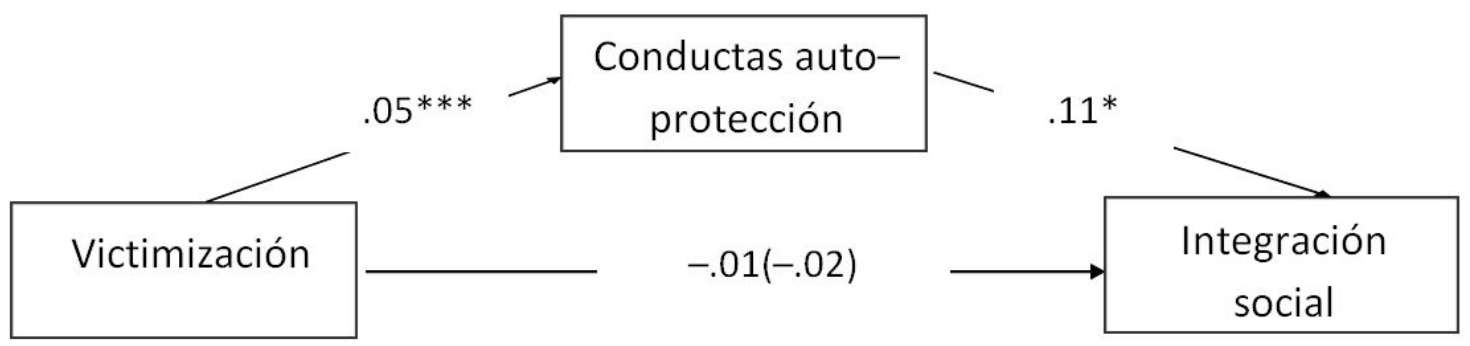

Figura 4. Efecto de la victimización sobre la integración social, reforzado a través de las conductas de auto-protección. En la figura se muestran los coeficientes de regresión no estandarizados. Además, se muestra el coeficiente del efecto total y, entre paréntesis, el coeficiente del efecto directo de la victimización sobre la actualización social. ${ }^{\star} p<.05 .{ }^{* \star} p<.01 .{ }^{* \star *} p<.001$.

Con respecto a la coherencia social (ver figura 5), los análisis de regresión indican que la victimización es un predictor significativo de las conductas de auto-protección, dado que una mayor victimización se asocia a una mayor frecuencia de conductas de protección personal. Asimismo, estas se relacionan de manera negativa y significativa con la coherencia social. La victimización no es un predictor significativo de la coherencia, aun cuando se controla por las conductas de protección. Sin embargo, la prueba de efecto indirecto basada en 
el procedimiento bootstrap es significativa $\left(B_{\text {efecto indirecto }}=-0.01\right.$; Boot $D E=0.00$; IC95\% [-0.0176. -0.0030]). Por ende, las conductas de auto-protección, como variable supresora, aumentan la validez predictiva de la variable victimización en la coherencia social por su inclusión en la regresión, es decir, reducen la capacidad de entender qué sucede alrededor, de poder encontrarle sentido y lógica a la vida.

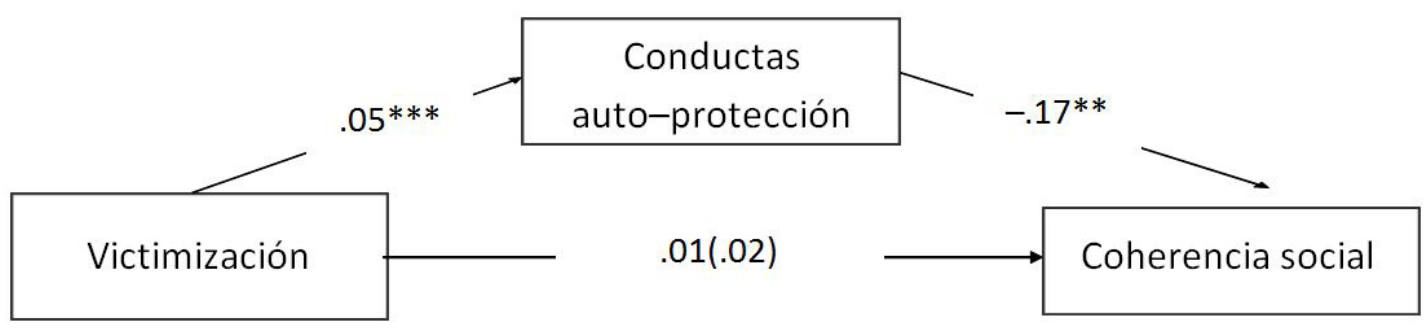

Figura 5. Efecto de la victimización sobre la coherencia social, reforzado por las conductas de auto-protección. En la figura se muestran los coeficientes de regresión no estandarizados. Además, se muestra el coeficiente del efecto total y, entre paréntesis, el coeficiente del efecto directo de la victimización sobre la actualización social. ${ }^{\star} p<.05 .{ }^{* \star} p<.01 .{ }^{* \star} p<.001$.

Como puede observarse en la figura 6 , la victimización no predice la actualización social, aun cuando se controlan las conductas de auto-protección, siendo el efecto directo no significativo en este modelo. Sin embargo, las pruebas de efecto indirecto basada en el procedimiento bootstrap es significativa $\left(B_{\text {efecto indirecto }}=\right.$ -0.01 ; Boot $D E=0.00$; IC95\% [-0.0207. -0.0028]). Esto quiere decir que una mayor victimización, asociada a conductas de auto-protección, conduce a menores niveles de actualización. Así, los participantes son más pesimistas respecto al futuro y las posibilidades de crecimiento y progreso social.

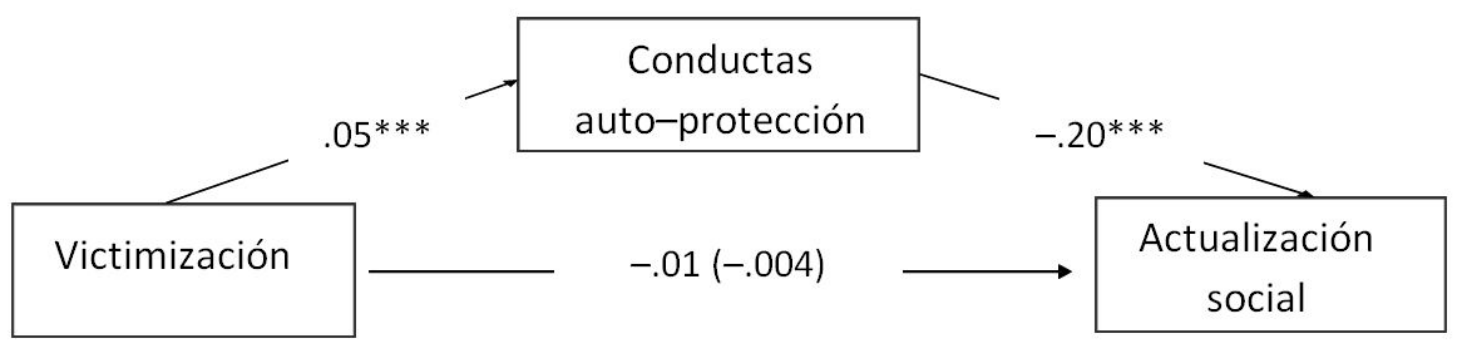

Figura 6. Efecto de la victimización sobre la actualización social, mediado por las conductas de auto-protección. En la figura se muestran los coeficientes de regresión no estandarizados. Además, se muestra el coeficiente del efecto total y, entre paréntesis, el coeficiente del efecto directo de la victimización sobre la actualización social. ${ }^{\star} p<.05 .{ }^{* \star} p<.01 .{ }^{* \star \star} p<.001$. 
En lo que hace al clima emocional, se detectan efectos de mediación significativos tanto para el clima emocional positivo como para el negativo. Como muestran las figuras 7 y 8 , el efecto total de la exposición al delito sobre el clima emocional es significativo y negativo respecto al clima positivo, mientras que significativo y positivo sobre el clima negativo. Esto es, una mayor victimización reduce la percepción del clima emocional positivo a la vez que aumenta la percepción del clima negativo. Estos efectos son significativamente menores al incluir en el modelo las conductas de auto-protección como mediadores, lo que confirma la hipótesis concerniente a los efectos indirectos de la victimización al delito a través de las conductas de auto-protección. Por un lado, los análisis de boostrap muestran un efecto indirecto de la victimización, sobre el clima emocional positivo, mediado por las conductas de protección personal $\left(B_{\text {efecto indirecto }}=-0.01\right.$; Boot $D E=0.00 ; \mathrm{IC} 95 \%$ [-0.0217. -0.0055]). Esto indica que los efectos negativos de la victimización sobre la percepción del clima positivo en parte se explican por una mayor presencia de conductas de auto-protección en quienes presentan una mayor exposición al delito, en comparación con los menos expuestos o no afectados. Se sostiene que estos efectos se explican sólo en parte por las conductas dado que el coeficiente de la victimización en el clima disminuye cuando se controlan las conductas, pero aun así sigue siendo significativo. Por otro lado, los análisis de boostrap muestran un efecto indirecto de la victimización sobre el clima negativo mediado por estas conductas $\left(B_{\text {efecto indirecto }}=0.02\right.$; Boot $D E=0.00$; IC95\% [0.0074. 0.0290]). De esta forma, el efecto positivo de haber sido víctima directa e indirecta de algún delito sobre la percepción del clima emocional negativo, se explica a través de las conductas. La mediación es parcial ya que el coeficiente de la victimización en el clima negativo disminuye cuando se controla el aspecto conativo pero, aunque menor, sigue siendo significativo - como ocurre en el caso del clima positivo.

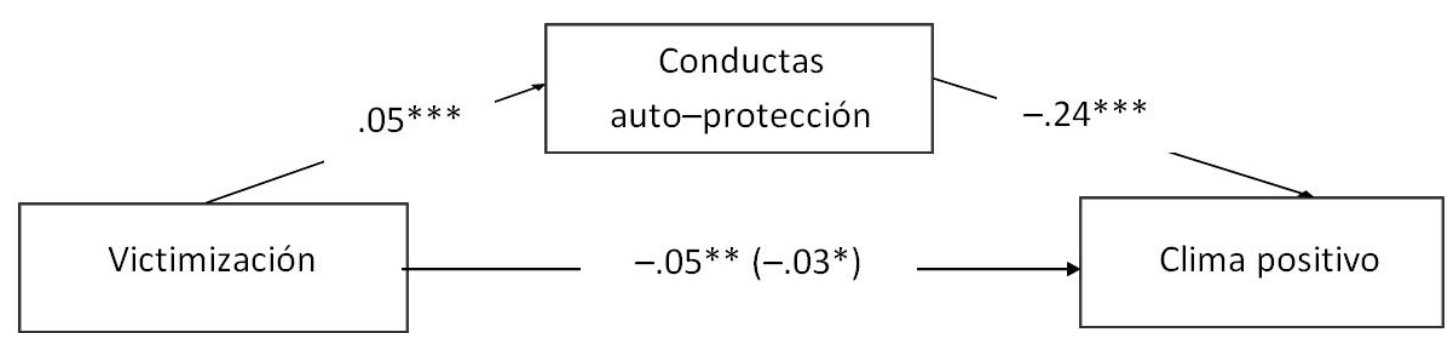

Figura 7. Efecto de la victimización sobre el clima emocional positivo, mediado por las conductas de auto-protección. En la figura se muestran los coeficientes de regresión no estandarizados. Además, se muestra el coeficiente del efecto total y, entre paréntesis, el coeficiente del efecto directo de la victimización sobre la actualización social.

$$
{ }^{\star} p<.05 .{ }^{* *} p<.01 .{ }^{* *} p<.001 .
$$




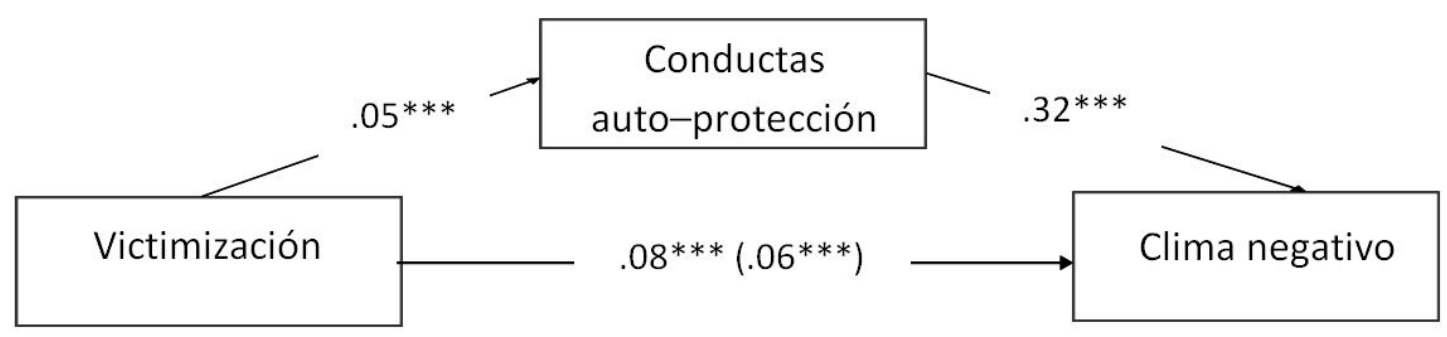

Figura 8. Efecto de la victimización sobre el clima emocional negativo, mediado por las conductas de auto-protección. En la figura se muestran los coeficientes de regresión no estandarizados. Además, se muestra el coeficiente del efecto total y, entre paréntesis, el coeficiente del efecto directo de la victimización sobre la actualización social.

$$
{ }^{\star} p<.05 .{ }^{\star \star} p<.01 .{ }^{* \star \star} p<.001 .
$$

\section{Discusión}

Los hallazgos obtenidos en el estudio permitieron verificar que tanto el riesgo percibido como el miedo al delito se asocian en menor medida y con menos intensidad al bienestar social, y más a la percepción del clima emocional. Los datos mostraron que tanto el riesgo percibido como el miedo al delito se asocian negativamente a la actualización social, y que este último también lo hace con la aceptación social. De esta forma, se deteriora tanto la evaluación de otras personas, al no considerarlas ni honestas ni confiables, como de la sociedad en su conjunto dado que se pierde la confianza en el progreso y bienestar que beneficie a todos.

Por su parte, las percepciones de inseguridad se relacionaron de manera negativa con el clima emocional positivo, y de manera positiva con el clima emocional negativo. Estos resultados corroboran lo planteado por de Rivera y Páez (2007) acerca de que la seguridad personal está asociada a las redes de apoyo de la persona y a la relativa ausencia de un clima negativo. Asimismo, que los países con climas emocionales más saludables presentan niveles uniformes de seguridad humana dado que hay menos diferencias entre las comunidades en donde las personas habitan. Ruiz y Turcios (2009) también encuentran que cuanto mayor es el temor y la probabilidad estimada de victimización, más negativamente se percibe el clima emocional y más saliente es la observancia de problemas sociales en el entorno.

Vanderveen (2006), a su vez, señala que la percepción de inseguridad erosiona la salud pública en términos de bienestar y calidad de vida, elevándose los niveles de aislamiento y exclusión social. Así, la percepción subjetiva de la inseguridad genera desorganización social, falta de cohesión y aumento del anonimato, llevando a una mayor desconfianza mutua y mayor propensión al temor (Bergman \& Kessler, 2008; Koonings \& Krujit, 2007; Ruiz, 2007), y al 
deterioro de los lazos comunitarios. Como sostienen Míguez e Isla (2010), la fragmentación social corroe la credibilidad y la confianza en las instituciones, contribuyendo al aumento del temor al delito. Por su parte, Smulovitz (2006) indica que la percepción de inseguridad es mayor entre aquellos sectores en los que el comportamiento anómico de las instituciones estatales es más alto y en las personas que más desconfían de las instituciones del Estado encargadas de la seguridad. En este punto, los estudios empíricos (Fleitas, Lodola, \& Flom, 2014; Kessler, 2009; Muratori \& Zubieta, 2013; Visser, Scholte, \& Scheepers, 2013) muestran que la corrupción, la desconfianza y el temor a la policía, la violencia institucional, la insatisfacción con la justicia y la falta de confianza social en general, predicen mayores niveles de miedo al delito y percepción de riesgo.

En función de estos resultados, y en coherencia con otros estudios (Ruiz, 2007; Ruiz \& Turcios, 2009) es relevante fomentar una línea de investigación que abarque la integración social y el sentido de comunidad mediante la participación en actividades comunitarias, a fin de disminuir las percepciones de peligrosidad y el miedo en el vecindario.

En lo que hace a las conductas de auto-protección, se verificó a estas como el indicador de inseguridad más asociado al bienestar social, en el sentido de que su aumento disminuye los niveles de aceptación, actualización y de coherencia, o viceversa, cuanto peores son las actitudes hacia otras personas como hacia la sociedad en su conjunto, dado que no se percibe que haya ni oportunidad de crecimiento ni lógica ni sentido, se limitan más las conductas a fin de protegerse contra el peligro. De esta forma, y conforme a Keyes (1998), una persona con menor coherencia social va a tener una peor adaptación a circunstancias impredecibles y a eventos. Los hallazgos corroboraron así lo encontrado por Lorenc et al. (2012) que muestran que las precauciones diarias que las personas llevan a cabo limitan la actividad de manera considerable, al punto de tener que reorganizar el estilo de vida a fin de evitar la exposición al riesgo. En este sentido, las conductas evitativas afectan el bienestar de las personas en dos sentidos: limitando la interacción personal, lo que lleva a una peor salud mental, y restringiendo la actividad física, lo que produce un deterioro en la salud física. A su vez, Ferraro (1995) plantea que la restricción de la conducta, o el cambio de rutina en la actividad, aumenta el cinismo acerca del orden social y la predicción del riesgo, mientras que Doran y Burgess (2012) señalan que las distintas formas de auto-protección producen un impacto y deterioro no sólo a nivel individual sino también a nivel comunitario.

Por último, los análisis mediacionales arrojaron resultados interesantes. Si bien los datos evidencian que la victimización no predice el bienestar social, si lo hace cuando intervienen la percepción de riesgo y las conductas de auto-protección. Es decir, el hecho objetivo de una mayor exposición al delito no genera cambios en la 
evaluación de los participantes respecto de sus relaciones y su funcionamiento en la sociedad. Sin embargo, al estar mediado por la percepción de riesgo, se disminuye el optimismo respecto al futuro de la sociedad, la confianza en el progreso y en el potencial de crecimiento y desarrollo. Por su parte, las conductas de autoprotección como mediadoras de la relación de la victimización y el bienestar social, disminuyen el optimismo respecto al futuro de la sociedad y la capacidad de esta para generar crecimiento y desarrollo. Además, se detectaron efectos de supresión de las conductas de protección personal sobre la coherencia y la integración social. En este sentido se observa que, conforme a lo planteado por Lorenc et al. (2012) respecto al impacto de las conductas de protección en el bienestar de las personas, estas aumentan la validez predictiva de la victimización sobre la coherencia, reduciendo la capacidad de los participantes para entender lo que sucede alrededor, de poder encontrarle sentido y lógica a la vida. No obstante, se observó que estas conductas posibilitan un mayor sentimiento de pertenencia a partir del cual se construyen lazos sociales más fuertes con la familia, amigos, vecinos, etc.

Finalmente, si bien la victimización predice la percepción del clima emocional, la intervención del miedo al delito, la percepción de riesgo y las conductas de auto-protección como factores mediadores mejoran este poder predictivo. La mayor exposición al delito hace que el clima emocional positivo se reduzca y aumente el negativo, en parte por experimentar mayor miedo al delito, por sentir más riesgo y por llevar a cabo más conductas de protección personal.

Los resultados obtenidos permiten concluir que tanto la percepción de inseguridad como las conductas de auto-protección inciden en el deterioro de la percepción del contexto social. El delito, y su consecuente victimización, son hechos objetivos cuya resolución depende de políticas públicas que conciernen a múltiples instituciones. La eficacia de las intervenciones que se diseñen y apliquen depende también de la concepción que se tenga de la problemática, en este caso de la inseguridad. Contemplar sólo la dimensión objetiva deja de lado todo aquello que tiene que ver con la evaluación de las percepciones de inseguridad y de las conductas de auto-protección que, a su vez, están vinculadas con la victimización y afectan la percepción del contexto social. La dimensión subjetiva de la inseguridad, propia de una mirada psicosocial, aporta evidencia acerca de que los cambios en los comportamientos para ser efectivos deben implicar cambios a nivel de la cognición, es decir, en los valores y creencias que favorecen el bienestar individual y social, y la consecuente mediación emocional. Como plantea Kessler (2007), dado que las políticas para reducir el crimen no tienen efectos en la reducción del miedo al delito, es que se debe pensar en nuevas estrategias que se orienten también en reducir la percepción de inseguridad por el impacto que esta tiene en la vida cotidiana de las personas, comunidades y calidad de vida urbana. 
Por lo tanto, las estrategias de intervención deben influir en la percepción de inseguridad concretamente, a fin de promover la seguridad subjetiva como uno de los ejes de la calidad de vida urbana. Pero para poder influirlas, modificarlas, es necesario tenerlas en cuenta. Es en este sentido que este trabajo de investigación se propuso explorar e identificar algunos de los elementos críticos de la dimensión subjetiva de la inseguridad, para aportar a futuras intervenciones que tengan en cuenta las formas en que las personas interpretan y dan sentido a lo que ocurre en su mundo, a fin de crear comunidades más seguras.

La inseguridad es una de las mayores problemáticas sociales que hoy en día caracterizan a los ciudadanos argentinos. Es un problema social, actual y relevante que, si bien no es nuevo, su magnitud y consecuencias, sobre todo en lo subjetivo y en la percepción del contexto social, sí lo son, y, como bien señala Smulovitz (2006), la brecha entre la percepción de inseguridad y las condiciones objetivas de criminalidad y violencia alimenta la alienación social de los ciudadanos y disminuye la calidad de vida ciudadana.

\section{Referencias}

Bergman, M. \& Kessler, G. (2008). Vulnerabilidad al delito y sentimiento de inseguridad en Buenos Aires: determinantes y consecuencias. Desarrollo Económico, 48(190/191), 209-234.

Chía Chávez, E., Bilbao, M. A., Páez, D., Iraurgi, I., \& Beristain, M. (2011). La importancia de los eventos traumáticos y su vivencia: el caso de la violencia colectiva. En D. Páez Rovira, C. M. Beristain, J. L. González-Castro, N. Basabe Barañano, \& J. de Rivera (Eds.), Superando la violencia colectiva y construyendo cultura de paz (pp. 165-205). Madrid: Editorial Fundamentos.

Cohen, M. A. (2008). The effect of crime on life satisfaction. The Journal of Legal Studies, 37(S2), S325-S353. http://dx.doi.org/10.1086/588220

Conger, A. J. (1974). A revised definition for suppressor variables: A guide to their identification and interpretation. Educational Psychological Measurement, 34, 35-46. http://dx.doi.org/10.1177/001316447403400105

Davies, S. \& Hinks, T. (2010). Crime and happiness amongst heads of households in Malawi. Journal of Happiness Studies, 11(4), 457-476. http://dx.doi. org/10.1007/s10902-009-9152-7

de Rivera, J. \& Páez, D. (2007). Emotional climate, human security and cultures 
of peace. Journal of Social Issues, 63(2), 233-253. http://dx.doi.org/10.1111/ j.1540-4560.2007.00506.x

Delfino, G. I. (2009). Participación política y factores psicosociales: un estudio con estudiantes universitarios (Tesis doctoral inédita). Facultad de Psicología, Universidad de Buenos Aires, Buenos Aires.

Doran, B. J. \& Burgess, M. B. (2012). Putting fear of crime on the map. Nueva York: Springer. http://dx.doi.org/10.1007/978-1-4419-5647-7

Farrall, S. \& Lee, M. (2009). Critical voices in an age of anxiety. A reintroduction to the fear of crime. En M. Lee \& S. Farrall (Eds.), Fear of Crime. Critical voices in an age of anxiety (pp. 1-11). Nueva York: Routledge Cavendish. Taylor \& Francis Group.

Ferraro, K. F. (1995). Fear of Crime: Interpreting Victimization Risk. Nueva York: State University of New York Press.

Fleitas, D., Lodola, G., \& Flom, H. (2014). Delito y violencia en América Latina y el Caribe: Perfil de los países de la región. Buenos Aires: Asociación para el Análisis de Políticas Públicas.

Föhrig, A. (2006). Introducción. En J. Varat \& A. Garland (Eds.), Participación ciudadana y percepción de inseguridad en América Latina (pp. 1-3). Washington: Woodrow Wilson International Center for Scholars.

Franc, R., Prizmic-Larsen, Z., \& Kaliterna Lipovcan, L. (2012). Personal security and fear of crime as predictors of subjective well-being. En D. Webb \& E. Willis-Herrera (Eds.), Subjective well-being and security (pp. 45-67). Nueva York: Springer Science. http://dx.doi.org/10.1007/978-94-007-2278-1_4

Freeman, R. B. (1999). The economics of crime. En O. Ashenfelter \& D. Card (Eds.), Handbook of labor economics (pp. 3529-3571). Amsterdam: North Holland Publishers.

Hanslmaier, M. (2013). Crime, fear and subjective well-being: How victimization and street crime affect fear and life satisfaction. European Journal of Criminology, 10(5), 515-533. http://dx.doi.org/10.1177/1477370812474545

Hanson, R. F., Sawyer, G. K., Begle, A. M., \& Hubel, G. S. (2010). The impact of crime victimization on quality of life. Journal of Traumatic Stress, 23(2), 189-197.

Jackson, J. (2009). A psychological perspective on vulnerability in the 
fear of crime. Psychology, crime and law, 15(4), 365-390. http://dx.doi. org $/ 10.1080 / 10683160802275797$

Jackson, J. \& Stafford, M. (2009). Public health and fear of crime: A prospective cohort study. British Journal of Criminology, 49, 832-847. http://dx.doi. org/10.1093/bjc/azp033

Kessler, G. (2007). Miedo al crimen. En A. Isla (Ed.), En los márgenes de la ley (pp. 69-99). Buenos Aires: Paidós.

Kessler, G. (2009). El sentimiento de inseguridad. Sociología del temor al delito. Buenos Aires: Siglo veintiuno editores.

Kessler, G. (2012). Delito, sentimiento de inseguridad y políticas públicas en la Argentina del siglo XXI. En Betancourt, J. A. (Ed.), La inseguridad y la seguridad ciudadana en América Latina (pp. 19-40). Buenos Aires: CLACSO.

Keyes, C. (1998). Social well-being. Social Psychology Quarterly, 61(2), 121-140. http://dx.doi.org/10.2307/2787065

Koonings, K. \& Krujit, D. (2007). Fractured cities: social exclusion, urban violence and contested spaces in Latin America. London: Zed Books.

Kuroki, M. (2013). Crime victimization and subjective well-being: Evidence from happiness data. Journal of Happiness Studies, 14, 783-794. http://dx.doi. org/10.1007/s10902-012-9355-1

Lorenc, T., Clayton, S., Neary, D., Whitehead, M., Petticrew, M., Thomson, H., ...Renton, A. (2012). Crime, fear of crime, environment, and mental health and well-being: Mapping review of theories and casual pathways. Health and Place, 18, 757-765. http://dx.doi.org/10.1016/j.healthplace.2012.04.001

Maslow, A. H. (1954/1987). Motivation and personality (3rd ed.). New York: Harper\&Row.

Míguez, D. \& Isla, A. (2010). Entre la inseguridad y el temor. Instantáneas de la sociedad actual. Buenos Aires: Paidós.

Moreno, C. (2014). Cultura democrática, confianza institucional y vida ciudadana (Informe anual Año IV). Recuperado del sitio de Internet de la Universidad Católica Argentina, Observatorio de la Deuda Social Argentina: http://www. uca.edu.ar/uca/common/grupo68/files/2014-Observatorio-BarometroDeuda-Social-Cap5.pdf 
Morrall, P., Marshall, P., Pattison, S., \& Macdonald, G. (2010). Crime and health: a preliminary study into the effects of crime on the mental health of UK university students. Journal of Psychiatric and Mental Health Nursing, 17, 821-828. http://dx.doi.org/10.1111/j.1365-2850.2010.01594.x

Muratori, M., Fernández, O., Bombelli, J. I., \& Zubieta, E. M. (2014). Anomia, bienestar social, percepción de control y confianza institucional. En E. Zubieta, J. Valencia \& G. Delfino (Eds.), Psicología Social y Política: procesos teóricos y estudios aplicados (pp. 393-410). Buenos Aires: EUDEBA.

Muratori, M. \& Zubieta, E. (2013). Miedo al delito y victimización como factores influyentes en la percepción del contexto social y clima emocional. Boletín de Psicología, 109, 7-18.

Muratori, M. \& Zubieta, E. (2016). Adaptación de la Escala de Bienestar Social al contexto argentino. Manuscrito en preparación.

Páez, D. \& Asún, D. (1994). Emotional climate, mood and collective behaviour: Chile 1973-1990. En H. Riguelme (Ed.), Era in twilight. Friburg (pp. 56-80). Bilbao: Foundation for children/Instituto Horizonte.

Páez, D., Ruiz, J. I., Gailly, O., Kornblit, A., Wiesenfeld, E., \& Vidal, C. M. (1997). Clima emocional: una investigación transcultural. Revista de Psicología Social, 1 (12), 79-98. http://dx.doi.org/10.1174/021347497320892045

Páez, D., Morales, J. F., \& Fernández, I. (2007). Las creencias básicas sobre el mundo social y el yo. En J. F. Morales, M. C. Moya, E. Gaviria \& I. Cuadrado (Eds.), Psicología Social (pp. 195-211). Madrid: McGraw-Hill.

Pegoraro, J. S. (2002). Las políticas de seguridad y la participación comunitaria en el marco de la violencia social. En R. Briceño-León (Ed.), Violencia, Sociedad y Justicia en América Latina (pp. 29-56). Buenos Aires: CLACSO.

Preacher, K. J., \& Hayes, A. F. (2008). Asymptotic and resampling strategies for assessing and comparing indirect effects in multiple mediator models. Behavior Research Methods, 40, 879-891. http://dx.doi.org/10.3758/BRM.40.3.879

Rottenbacher de Rojas, J., Amaya López, L., Genna Miyahira, K., \& Pulache Páez, M. (2009). Percepción de inseguridad ciudadana y su relación con la ideología política en una muestra de habitantes de la ciudad de Lima. Revista Española de Investigación Criminológica, 7, 1-22.

Ruiz, J. I. (2007). Procesos sociales relacionados con el miedo al crimen, la 
satisfacción con la policía y la victimización: El caso de la cultura ciudadana. International E-journal of criminal Science, l(1). Recuperado de http://www. ehu.eus/ojs/index.php/inecs/article/view/17/19

Ruiz, J. I., \& Turcios, L. A. (2009). Percepción de inseguridad, victimización y cultura ciudadana: sus relaciones en cinco contextos iberoamericanos. Pensamiento psicológico, 6(13), 193-202.

Schwartz, S. H. (2001). ¿Existen aspectos universales en la estructura y contenido de los valores humanos? En M. Ros \& V. Gouveia (Eds.), Psicología Social de los Valores Humanos (pp. 53-76). Madrid: Biblioteca Nueva.

Smulovitz, C. (2006). Seguridad ciudadana: comparando intervenciones en seis ciudades. En J. Varat \& A. Garland (Eds.), Participación ciudadana y percepción de inseguridad en América Latina (pp. 29-40). Washington: Woodrow Wilson International Center for Scholars.

Staubli, S., Killias, M., \& Frey, B. S. (2014). Happiness and victimization: An empirical study for Switzerland. European Journal of Criminology, 11(1), 57-72. http://dx.doi.org/10.1177/1477370813486866

Sulemana, I. (2015). The effect of fear of crime and crime victimization on subjective well-being in Africa. Social Indicators Research, 121, 849-872. http://dx.doi.org/10.1007/s11205-014-0660-4

Techio, E., Zubieta, E., Páez, D., de Rivera, J., Rimé, B., \& Kanyangara, P. (2011). Clima emocional y violencia colectiva: el estado de la cuestión e instrumentos de medición. En D. Páez Rovira, C. M. Beristain, J. L. González-Castro, N. Basabe Barañano \& de Rivera, J. (Eds.), Superando la violencia colectiva y construyendo cultura de paz (pp. 105-150). Madrid: Editorial Fundamentos.

Vanderveen, G. (2006). Interpreting fear, crime, riskand unsafety. Conceptualisation and measurement. Den Haag: Boom Juridische uitgevers.

Visser, M., Scholte, M., \& Scheepers, P. (2013). Fear of crime and feelings of unsafety in European countries: Macro and micro explanations in crossnational perspective. The Sociological Quarterly, 54, 278-301. http://dx.doi. org/10.1111/tsq. 12020

Vozmediano, L. (2010). Miedo al delito y sostenibilidad urbana: análisis ecológico, propuestas de medición y transferencia de resultados (Tesis doctoral inédita). Universidad del País Vasco, San Sebastián. 
Vozmediano, L., Vergara, A. I., \& San Juan, C. (2010). El estudio científico del miedo al delito: algunas reflexiones sobre un fenómeno urbano, mediático y político. International e-Journal of Criminal Science, 2(4). Recuperado de http://www.ehu.eus/ojs/index.php/inecs/article/view/924/678

Zubieta, E. M., Muratori, M., \& Mele, S. (2012). Bienestar, clima emocional, percepción de problemas sociales y confianza. Anuario de Investigaciones, 19, 97-106. 
